\title{
Integrated System for the Rapid Polycyclic Aromatic Hydrocarbons Extraction from Aqueous Samples and Their Consecutive Thermal Desorption Prior to Gas Chromatography Analysis
}

\author{
Florence Ricoul1,2 (ํ); Roland Pelisson1,2, Bruno Fain1,2, Sévérine Vignoud1,2 \\ ${ }^{1}$ University Grenoble Alpes, Grenoble, France \\ ${ }^{2}$ CEA, LETI, MINATEC Campus, Grenoble, France \\ Email: ^florence.ricoul@cea.fr
}

How to cite this paper: Ricoul, F., Pelisson, R., Fain, B. and Vignoud, S. (2020) Integrated System for the Rapid Polycyclic Aromatic Hydrocarbons Extraction from Aqueous Samples and Their Consecutive Thermal Desorption Prior to Gas Chromatography Analysis. American Journal of Analytical Chemistry, 11, 75-89.

https://doi.org/10.4236/ajac.2020.112006

Received: October 23, 2019

Accepted: February 8, 2020

Published: February 11, 2020

Copyright $\odot 2020$ by author(s) and Scientific Research Publishing Inc. This work is licensed under the Creative Commons Attribution International License (CC BY 4.0).

http://creativecommons.org/licenses/by/4.0/

\begin{abstract}
This paper describes for the first time the extraction followed by thermal desorption of polycyclic aromatic hydrocarbons (PAHs) spiked water samples in a microfluidic silicon device. Thanks to the integration into an original system composed of a micropump, microvalves, and an optimized thermal management, the entire protocol is automated and combines the extraction, the drying and the desorption in less than 25 min before sending the sample to a GC-FID system. Repeatable recovery yields have been determined for $1 \mu \mathrm{g} / \mathrm{L}$ spiked water samples and the analysis of PAHs in a natural water spiked sample has been demonstrated without loss of performance compared to purified water samples. Compared to other extraction techniques, this system has the advantage of reduced footprint, reduced energy consumption and no solvent use.
\end{abstract}

\section{Keywords}

Microfluidic Device, Extraction, Thermal Desorption, PAH, Water

\section{Introduction}

Polycyclic aromatic hydrocarbons (PAHs) are known to present toxic, mutagenic and carcinogenic effects. They are ubiquitous present in the environment because of human activity [1]. PAHs detection is therefore a crucial concern for environment control and human health to avoid possible excessive exposure. 
The European Union and the United States Environmental Protection Agency have both formulated regulations for monitoring and controlling PAHs [2] [3], in particular in drinking water.

Numerous analyses have to be carried out daily in laboratories to ensure a good monitoring of water quality. Common analytical methods consist in several steps, namely a sampling step in the field, a transfer to the lab, an extraction step to purify and concentrate the analytes, a desorption step and finally an analytical step [4] [5]. Various extraction methods have been developed, including extraction on solid phases such as Solid Phase Extraction (SPE), Solid Phase Micro-Extraction (SPME) and Stir Bar Sorptive Extraction (SBSE) [6] [7]. The desorption step may be operated in liquid phase using solvent desorption or in gaseous phase using thermal desorption. The separation and detection of species may be achieved in liquid phase, using high-performance liquid chromatography (HPLC) coupled with fluorescence detection (FLD) or mass spectrometry (MS). Alternatively, this step may be operated by gas chromatography (GC) combined with MS or Flame Ionization detector (FID). A lot of developments are still in progress to improve the sensitivity or the through-put of such methods, either by working on the development of more efficient matrices for the extraction [8] [9] or by introducing new methodologies [10]-[14]. The automation of such analyzes is also investigated, yet it remains expensive, cumbersome and time-consuming.

In parallel to such lab techniques, some portable systems have been developed recently for field analysis. Portable systems avoid transport and storage of the analytes that may induce biases on the results [15]. The portable GC-MS Torion ${ }^{\circledast}$ by Perkin-Elmer uses SPME fibers for the analysis of compounds in water matrix. The Hapsite ${ }^{\circledast}$ by Inficon uses the dedicated SituProbe ${ }^{\mathrm{TM}}$ technology based on purge and trap principle for the VOCs analysis in water samples. In both cases, the systems need some manual operation for the samples analysis and are not adapted for continuous monitoring.

Over the last past decade, several research teams have also explored the microfluidic approach for sample preparation and preconcentration in order to benefit from portability, low sample volume capability, low reagent consumption, low fabrication cost and potential parallelization [16]. They have either used SPE in particular within centrifugal microfluidic device [17] [18] or Liquid-Liquid Extraction [19] [20]. The full integration of the devices has not been demonstrated yet and they require some consumables (solvent) or present weak sensitivity for PAHs.

In previous works, our team has demonstrated the rapid extraction of PAHs using a microfluidic device based on a glass/silicon chip [21] [22]. In particular, we demonstrated that porous SiOCH type adsorbent phase, deposited by a collective, very reproducible, process, could be used, allowing extraction yields even better than the classical PDMS polymeric phase [22]. The desorption was performed by liquid desorption, using a solvent. In fact fast extraction has several 
advantages: it avoids the possible degradation of the samples (adsorption on vial walls, etc.) as described above, allows a shorter response time so in the case of a pollution, protective measures could be applied as soon as possible, permits higher analysis through-put which would allow more samples analysis, and reduced costs. In the present study, we show that thermal desorption can also be used to transfer the analytes to the analytical system, in this case a GC/FID. Indeed thermal desorption is a good alternative to liquid desorption: it does not use a consumable (solvent) and is more suitable for integration, automation of the protocol and coupling with GC. As already mentioned in the literature for gas sample analysis [23] [24] [25] [26], it is very advantageous to use silicon for the realization of microcomponents needing to be heated because of its very good thermal conductivity. It allows to obtain very fast heating ramps and also a relatively fast cooling without fans or thermoelectric materials. In addition, the power consumption is tiny, about a few watts compared to thousands watts for a standard GC oven. In the present work, we have also developed an original system architecture which allows to carry out the sample extraction, the drying, the desorption and the analysis without any manual intervention, in a completely automated way. Our module can interface with any type of GC device, in particular with portable GC devices that could enable in-situ analysis of PAHs in natural waters. The current study presents first the validation of the on-chip thermal desorption of PAHs after extraction. Then the extraction yields obtained for purified water samples spiked with 16 PAHs using the novel integrated system are given and finally the validation on spiked natural water samples is reported.

\section{Experimental Part}

\subsection{Standards and Reagents}

The 16 PAHs targeted by the United State Environmental Protection Agency since 1976 are considered in this study. A standard mix of the 16 PAHs at 10 $\mu \mathrm{g} / \mathrm{mL}$ in methanol was purchased from Sigma-Aldrich (USA) to prepare standard and spiked water samples. It contains naphthalene (NAP), acenaphtylene (ACL), acenaphtene (ACE), fluorene (FLR), phenanthrene (PHE), anthracene (ANT), fluoranthene (FTN), pyrene (PYR), benz(a)anthracene (B(a)A), chrysene $(\mathrm{CHR})$, benzo(b)fluoranthene $(\mathrm{B}(\mathrm{b}) \mathrm{F})$, benzo[k]fluoranthene $(\mathrm{B}(\mathrm{k}) \mathrm{F})$, benzo(a)pyrene (B(a)P), indeno(1,2,3-cd)pyrene (IND), dibenzo(a, h) anthracene $(\mathrm{D}(\mathrm{h}) \mathrm{A})$ and benzo(g, h, i)perylene $(\mathrm{B}(\mathrm{ghi}) \mathrm{P})$. Number of rings, water-octanol coefficients and molecular weight of the 16 PAHs are given in Table S1 in Supplementary Material.

Purified water was obtained from a MilliQ system (Millipore, USA). Syringe filters with $0.45 \mu \mathrm{m}$ polyethersulfone membranes (PALL, Port Washington, NY, USA) were used for filtering the natural environmental water samples from Isère river.

\subsection{Silicon/Glass Microchip Fabrication}

Silicon/glass microfluidic chips (Figure 1) were manufactured from $200 \mathrm{~mm}$ sil- 
icon wafers using standard micro-technology techniques such as photolithography and Deep Reactive Ion Etching (DRIE) as described elsewhere [27]. The internal chamber contains rows of squared micro-pillars of $17 \mu \mathrm{m}$ side, $230 \mu \mathrm{m}$ height and a $10 \mu \mathrm{m}$ gap between pillars (Figure 1). External dimension of the chip is $30 \times 12 \mathrm{~mm}^{2}$. A $150 \mathrm{~nm}$ porous $\mathrm{SiOCH}$ thin layer was used as extraction phase and deposited by PECVD before glass top anodic bonding as described in [22]. Before chip dicing, a heating resistor ( $8.5 \mathrm{Ohms})$ and temperature probes (100 Ohms) were deposited on the back side by sputtering a thin Pt layer. Finally fused-silica capillaries (internal diameter $250 \mu \mathrm{m}$, outer diameter $360 \mu \mathrm{m}$ ) from Photon Lines (France) were glued to the inlets for fluidic connection.

\subsection{System Architecture and Protocols}

The system architecture is shown in Figure 2. The system contains a peristatic pump (model P625 from Instech), a pressure regulator (IQ + flow from Bronkhorst), two 3-way and one 2-way valves (the Lee Company), a power supply board (NV1-4G5TT), electronic modules (NI9263, NI9217, NI9403 and cDAQ9174 from National Instrument) and a homemade interface board. A fused silica "Y" connector from Supelco is used for the fluidic tee connection, whereas $30 \mathrm{~cm}$ long fused-silica capillary (internal diameter $250 \mu \mathrm{m}$, outer diameter $360 \mu \mathrm{m}$ ) is

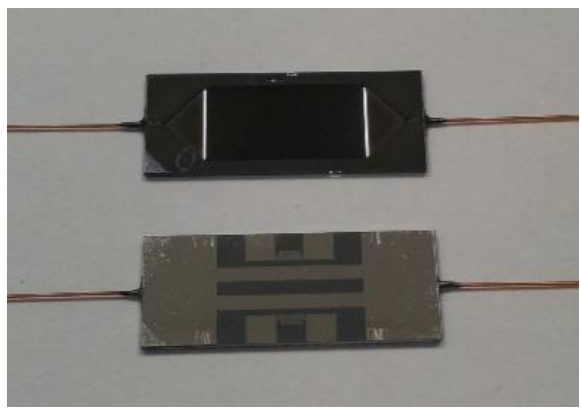

Figure 1. Picture of the glass/silicon microfluidic chips with glued capillary tubing: front side, view of the chamber with micro pillars; back side, view of the platinum heating resistance and temperature probes.

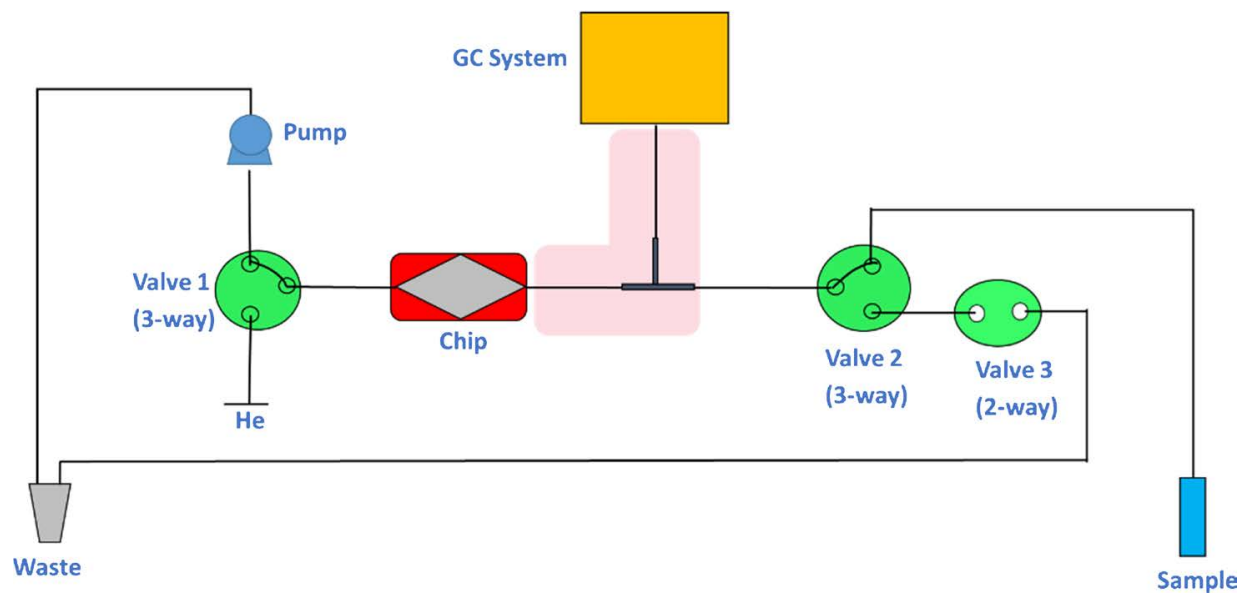

Figure 2. System diagram (pink zone = temperature controlled zone). 
used between the tee and the GC capillary column. The originality of the assembly lies in the fact that the gaseous sample of PAHs never passes through a valve and that the transfer line and the fluidic tee through which PAHs pass after desorption are both heated using powered carbon fibers wrapped around the connections to avoid cold spots. Thus, the valves do not have to be regulated in temperature which makes it possible to reduce the power consumption and the analysis times since no long cooling time between each analysis is required.

All the system components are assembled in a $35 \mathrm{~cm} \mathrm{(l)} \times 35 \mathrm{~cm} \mathrm{(w)} \times 27 \mathrm{~cm}$ (h) module as shown in Figure 3. The system is connected through USB with a laptop for device control with a homemade Labview application. In particular chip and transfer line temperatures are regulated using PID Labview function and the feedback of the temperature measurements of the chip and along the transfer line.

The system operation is divided in 4 modes (Figure 4): in the extraction mode, the pump is activated and valves are configured so that sample is pumped through the microchip at $1 \mathrm{~mL} / \mathrm{min}$. In the drying mode, pump is stopped, helium pressure is set to 1 bar and valves are switched so that the gas flow is blowing
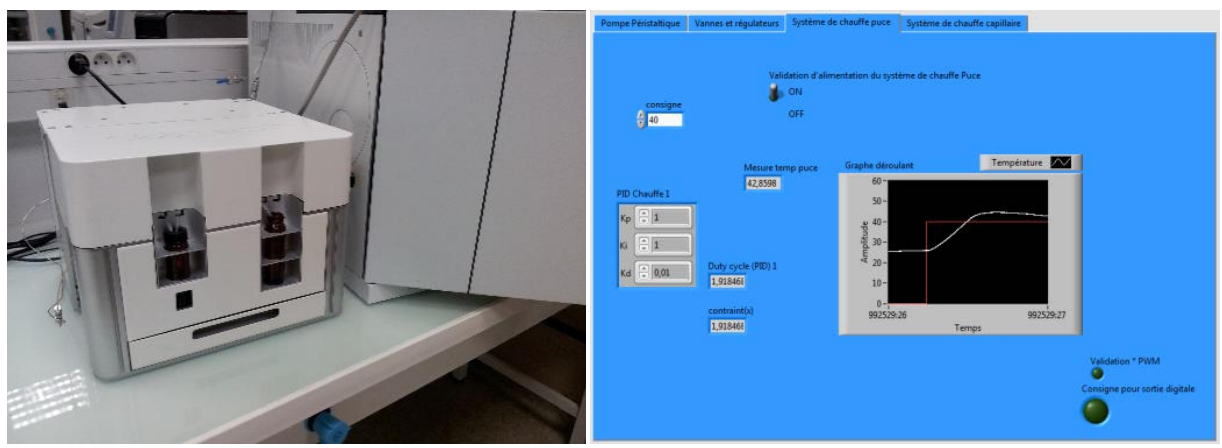

Figure 3. Left: picture of the prototype; Right: screenshot of the Labview application interface.

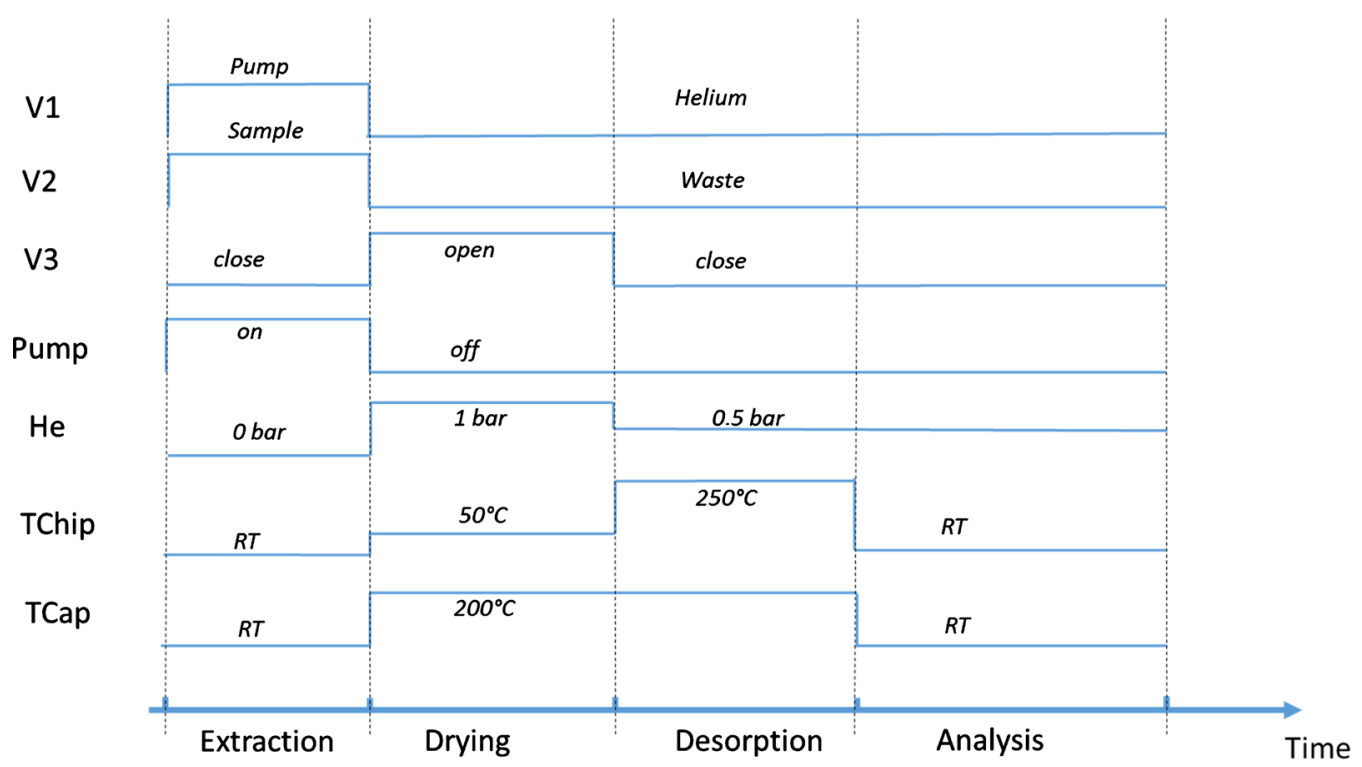

Figure 4. Timing diagram. 
through the chip towards waste for $10 \mathrm{~min}$. To ease the drying, the chip temperature is set to $50^{\circ} \mathrm{C}$ and the capillary/fluidic tee temperature is set to $200^{\circ} \mathrm{C}$. The drying of the chip under a carrier gas flow at mild temperature before the thermal desorption removes residual excess water and avoids problems with premature degradation of the capillary column stationary phase or FID signal disturbance. Because of the difference in the fluidic impedance between the capillary towards waste and the capillary towards the GC column, there is a difference in pressure drop between the path to the waste and the one to GC column so that no water contamination is observed in the latter path.

During the desorption stage, valve 3 is closed, helium pressure is set to $0.5 \mathrm{bar}$ and chip temperature is set to $250^{\circ} \mathrm{C}$ for $5 \mathrm{~min}$. The temperature of $250^{\circ} \mathrm{C}$ is reached within $60 \mathrm{~s}$ with a $24 \mathrm{~V}$ power supply. Molecules that are desorbed from the chip are thus focused at the GC column inlet which is maintained at the GC oven temperature, i.e. $50^{\circ} \mathrm{C}$. Finally during the analysis mode, chip and capillary heating is stopped while maintaining helium pressure at 0.5 bar and valves configuration as in stage 3. GC analysis is operated using the thermal gradient described in the next session.

\subsection{Analytical Methods}

A $2 \mathrm{~m}$ long $5 \mathrm{MS}$ capillary column (Supelco) of $250 \mu \mathrm{m}$ internal diameter and 0.2 $\mu \mathrm{m}$ of phase was used. During analysis, the flow rate was measured at $1 \mathrm{~mL} / \mathrm{min}$. The column temperature was initially set at $50^{\circ} \mathrm{C}$, then increased to $180^{\circ} \mathrm{C}$ at $20^{\circ} \mathrm{C} / \mathrm{min}$, to $240^{\circ} \mathrm{C}$ at $10^{\circ} \mathrm{C} / \mathrm{min}$ and to $250^{\circ} \mathrm{C}$ at $5^{\circ} \mathrm{C} / \mathrm{min}$ and hold $1 \mathrm{~min}$ at this temperature. The temperature of the FID detector was set to $250^{\circ} \mathrm{C}$.

In the case of direct injection of the commercial mix for calibration procedure, the column was connected to the split/splitless injector. Liquid samples of $2 \mu \mathrm{L}$ were injected with a split ratio of 9.3 and a temperature of $250^{\circ} \mathrm{C}$.

Extraction yields were estimated by comparing peak areas obtained with the FID after the whole protocol (sample extraction/drying/desorption/analysis) and those measured from the direct injection of the commercial mix of 16 PAHs.

\section{Results and Discussion}

\subsection{Validation of the On-Chip Thermal Desorption and the Coupling with GC-FID}

In our previous studies we have shown that our microfluidic chips could extract efficiently within $20 \mathrm{~min}$ PAHs contains in $10 \mathrm{~mL}$ water samples, either by using PDMS extraction phase or SiOCH extraction phase [21] [22]. Desorption was performed using a solvent (acetonitrile) and the detection was done by HPLC-FLD. In this study we go further in the integration and development of a transportable system, using thermal desorption which does not use a consumable (solvent) and is more suitable for integration, automation of the protocol and coupling with GC.

An extraction rate of $1 \mathrm{~mL} / \mathrm{min}$ was used to extract the PAHs contained in 10 
$\mathrm{mL}$ water sample (i.e. extraction time of $10 \mathrm{~min}$ ) since no degradation of the recovery rate was observed, compared with the 20 min time of our previous studies. Extraction is thus rather fast compared to other usual solid phase extraction methods [11].

The relevance of on-chip thermal desorption was assessed by comparing the analysis of a $10 \mathrm{~mL}$ water sample spiked at $1 \mu \mathrm{g} / \mathrm{L}$ with 16 PAHs extracted on the chip and recovered by thermal desorption with the analysis of a commercial reference sample directly injected in the GC. In Figure 5 are shown the chromatogram obtained after the extraction of the water sample followed by drying and thermal desorption, as well as the reference chromatogram. Except for the most volatile PAH (Naphthalene), all PAHs peaks can be identified on the chromatogram corresponding to the extracted sample. Two pairs of PAHs (CHR and $\mathrm{B}(\mathrm{a}) \mathrm{A}$ as well as $\mathrm{B}(\mathrm{b}) \mathrm{F}$ and $\mathrm{B}(\mathrm{k}) \mathrm{F})$ are not resolved on both chromatograms. The use of a short 2 meter-long GC column, consistent with the perspective of portable miniaturized GC system, explains that not all peaks are resolved. Other peaks present in the first chromatogram are due to some interfering compounds also observed in the case of blank (pure water) samples as illustrated in Figure 6.

Some preliminary experiments have shown that a connection capillary and fluidic T temperature of $200^{\circ} \mathrm{C}$ is required but sufficient to keep all the PAHs in the gaseous phase until the separation column inlet. The desorption duration and chip temperature have respectively been set to 5 minutes and $250^{\circ} \mathrm{C}$ so that all the PAHs are well desorbed from the chip at one time: in Figure 7, the almost flat chromatogram confirms that desorption is complete at the first chip heating.
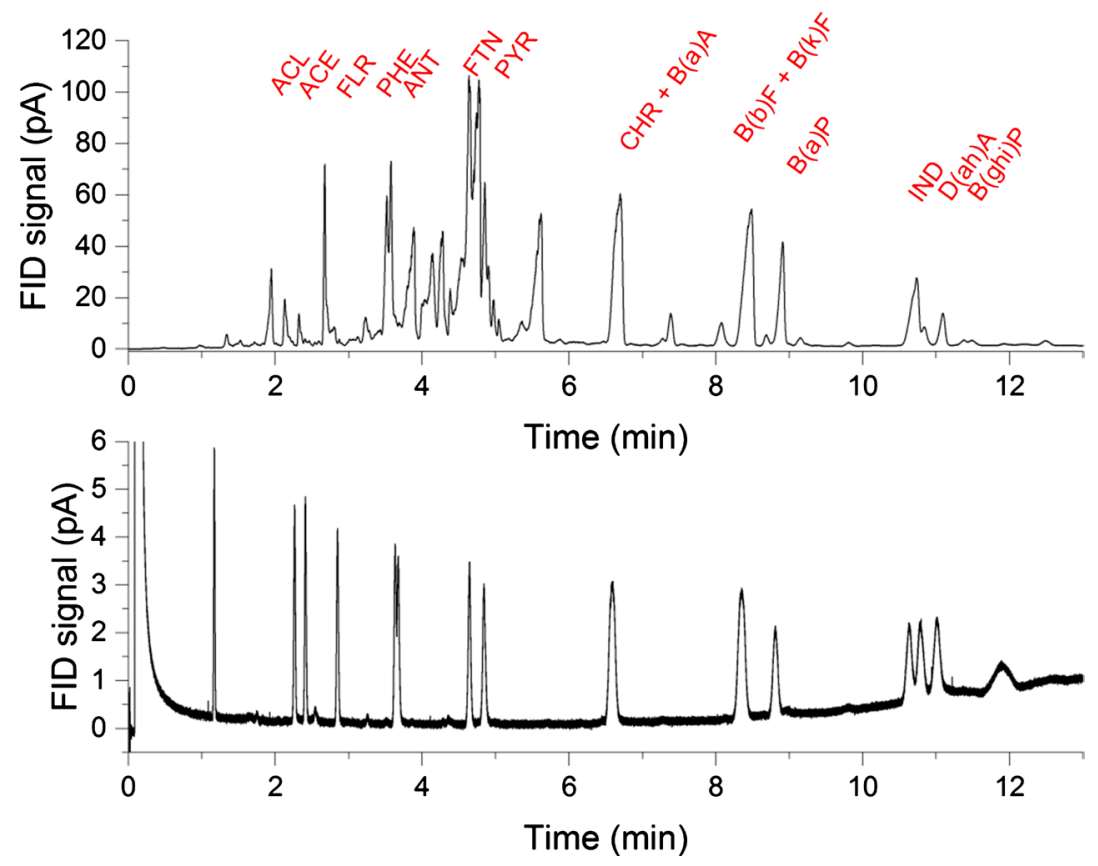

Figure 5. Top: chromatogram obtained after extraction of $10 \mathrm{~mL}$ of purified water spiked at $1 \mu \mathrm{g} / \mathrm{L}$ with the $16 \mathrm{PAHs}$ followed by thermal desorption; bottom: chromatogram obtained with the commercial mixture at $10 \mu \mathrm{g} / \mathrm{mL}$ in acetonitrile directly injected within the column. 


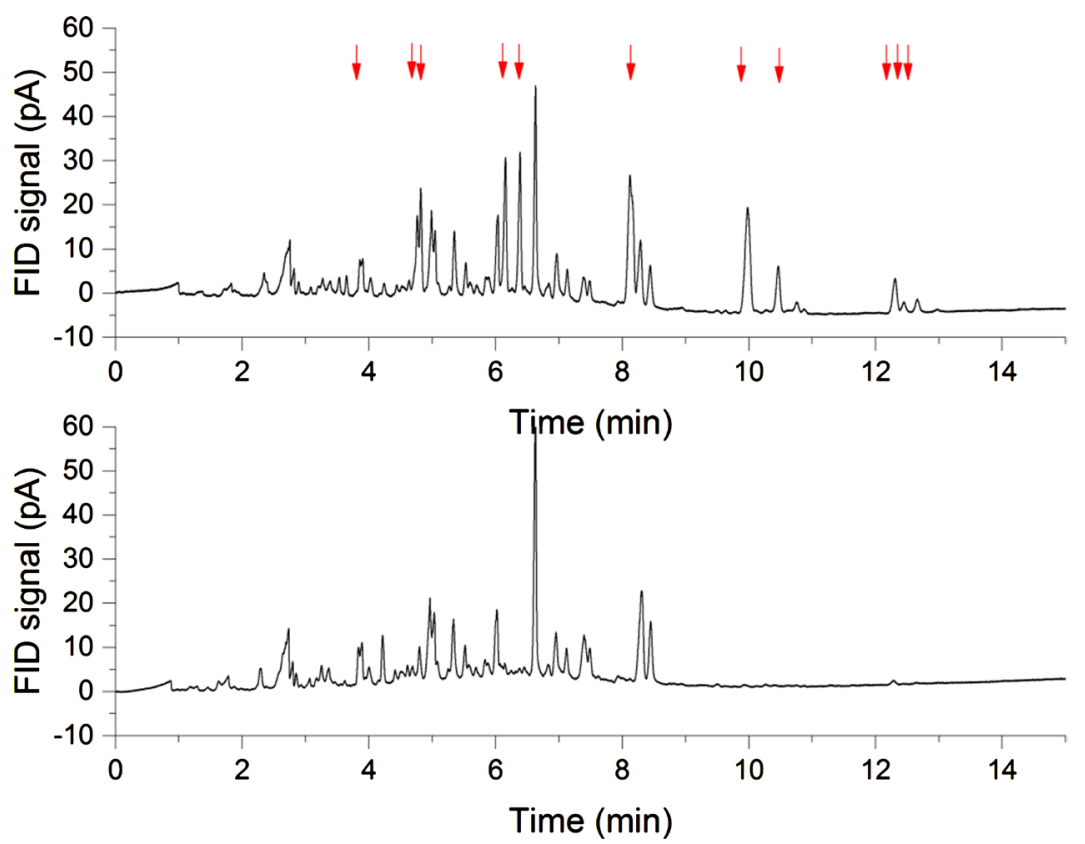

Figure 6. Top: chromatogram obtained for the extraction of $5 \mathrm{~mL}$ of purified water spiked with the 16 PAHs at $5 \mu \mathrm{g} / \mathrm{L}$; bottom chromatogram obtained for the extraction of 5 $\mathrm{mL}$ of purified water without spiking. (GC oven thermal gradient used in this figure was 2 min longer than the one used in Figure 5).

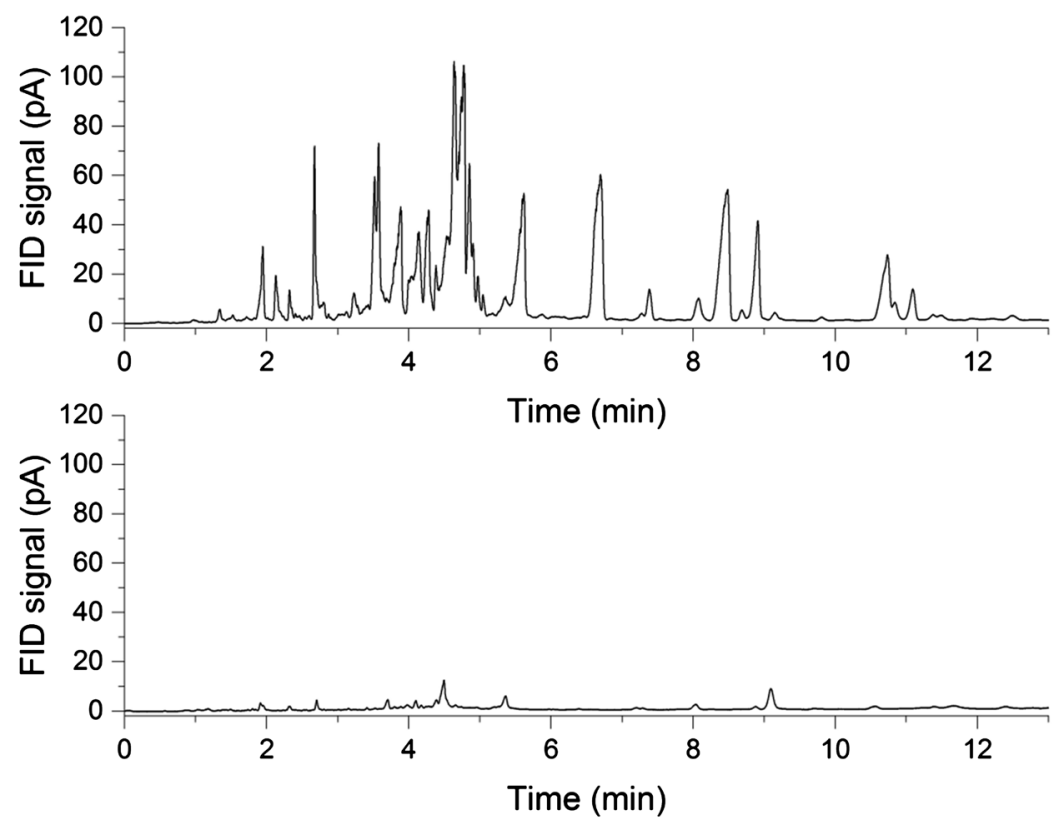

Figure 7. Top: chromatogram obtained for the extraction of $10 \mathrm{~mL}$ of purified water spiked with the $16 \mathrm{PAHs}$ at $1 \mu \mathrm{g} / \mathrm{L}$ (idem as in Figure 5); bottom: chromatogram obtained after the second desorption of the chip.

Moreover, we checked that no cross-contamination between the samples occurs. The blank samples were always clear, which means that there is no undesired adsorption along the system path.

These results prove that it is possible to carry out the extraction and the ther- 
mal desorption of PAHs on silicon-based chips before a GC-FID analysis with reduced time and energy consumption. The whole protocol, from extraction to desorption including the drying step, lasts only 25 minutes.

\subsection{Estimation of the Extraction Yields}

By comparing the peaks areas obtained after thermal desorption with those measured in the case of the direct injection of the commercial mix, one can deduce the extraction yield of our system. If two peaks are not resolved, the sum of the 2 PAHs quantities is taken into account. The extraction yield varies from $7.8 \% \pm 1.2 \%$ for FLR to $105.4 \% \pm 9.1 \%$ for $(\mathrm{CHR}+\mathrm{B}(\mathrm{a}) \mathrm{A})$, as detailed in Table 1 and Figure 8. Quite good repeatability of the protocol is observed, as reflected by the average Relative Standard Deviation (RSD) of $12 \%$ calculated on the basis of 4 analysis performed in the same conditions. These analyses have been carried out over 2 days. The chip-to-chip dispersion of the yields is also low (cf. Figure S1 in Supplementary Material). Besides we have observed that the same chip could be used for dozens of analyzes without loss of performance.

These yields are slightly lower than the ones deduced from our previous study using solvent desorption [22]. This could be explained by some losses of the PAHs molecules during our protocol, in particular during the chip drying, some of the PAHs going possibly to the waste path. Measurement protocol may be improved to increase the recovery yield of the most volatile PAHs (shorter extraction, drying or desorption times, use of more polar extraction phase). Yet, this would probably imply to lose some signal for the heavier PAHs. By running sequential analysis with different parameters, it should be possible to get responses for the whole range of PAHs with the same system.

Table 1. Average extraction yields and Relative Standard Deviation calculated from 4 different extractions of $10 \mathrm{~mL}$ of pure water sample spiked at $1 \mu \mathrm{g} / \mathrm{L}$ with the 16 PAHs on the same chip.

\begin{tabular}{ccc}
\hline PAH & Recovery (\%) & RSD (\%) \\
FLR & $10 \pm 2$ & 15 \\
PHE+ANT & $52 \pm 10$ & 20 \\
FTN & $65 \pm 4$ & 7 \\
PYR & $55 \pm 10$ & 18 \\
CHR + B(a)A & $64 \pm 6$ & 9 \\
B(b)F + B(k)F & $54 \pm 7$ & 13 \\
B(a)P & $49 \pm 7$ & 14 \\
IND & $43 \pm 5$ & 12 \\
D(ah)A & $21 \pm 1$ & 6 \\
B(ghi)P & $29 \pm 1$ & 5 \\
Mean & $44 \pm 5$ & 12
\end{tabular}




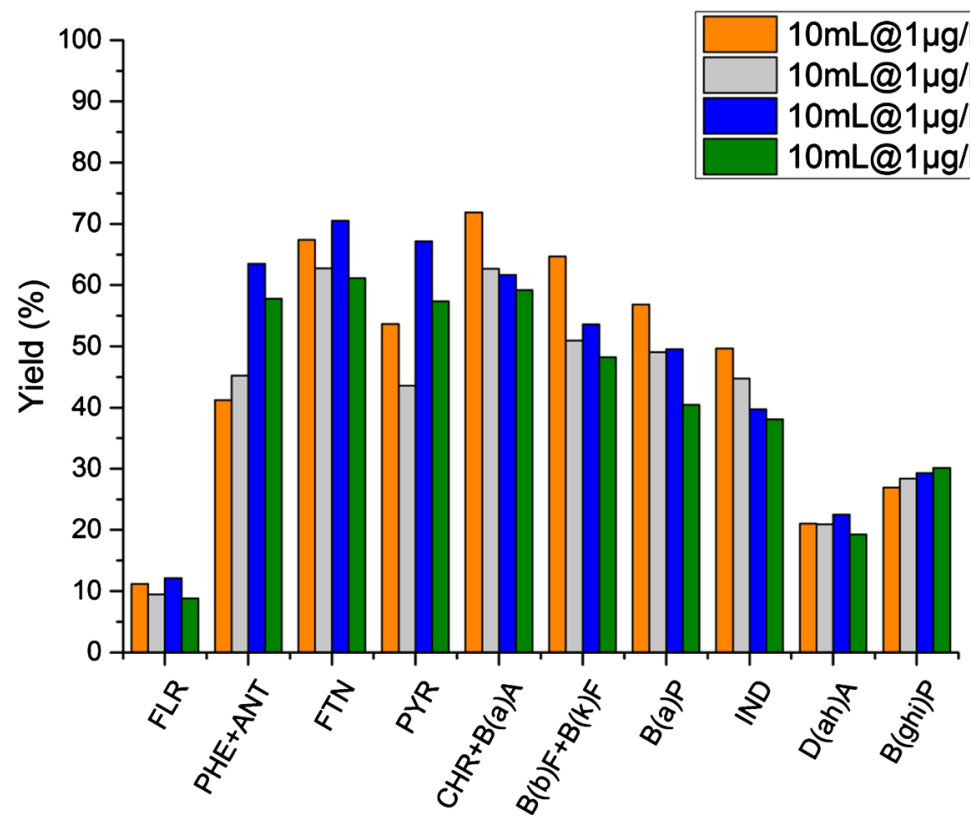

Figure 8. Extraction yield obtained for 4 different extractions of $10 \mathrm{~mL}$ of pure water sample spiked with the 16 PAHs at $1 \mu \mathrm{g} / \mathrm{L}$.

\subsection{Natural Water Samples Analysis}

It is known that dissolved organic matter present in environmental samples could affect the performance of the solid phase extraction [28] [29]. In order to check that our system could be used for the monitoring of such environmental samples, we performed the extraction of spiked natural water sample. As described in the experimental part, sample from river Isère was collected, filtered and spiked with the commercial PAHs solutions up to $5 \mu \mathrm{g} / \mathrm{L}$. We checked that no PAHs could be detected in the filtered river sample before the spiking step, either because they were not present in the sample, or because the concentrations were lower than the limit of detection of our system.

As seen in Figure 9, no large difference is observed between the recoveries in the case of purified water or natural water: heavier PAHs seem to get even better recoveries in the case of the natural water. This experiment therefore validates the use of our prototype for the extraction and desorption of PAHs in natural water samples prior to GC analysis.

\section{Conclusion}

This work presents the demonstration of on-chip extraction and thermal desorption of PAHs prior to GC analysis. Using a glass/silicon microfluidic device, functionalized with nanoporous $\mathrm{SiOCH}$ as the adsorbent phase, $10 \mathrm{~mL}$ PAHs samples are extracted within $10 \mathrm{~min}$ and thermally desorbed in less than $5 \mathrm{mi}$ nutes by a quick heating of the chip. The device has been embedded in an original system architecture with a micropump and microvalves which allow the complete automation of the extraction/desorption protocol and avoids any cold point with an optimal thermal management of the different parts. Our system 


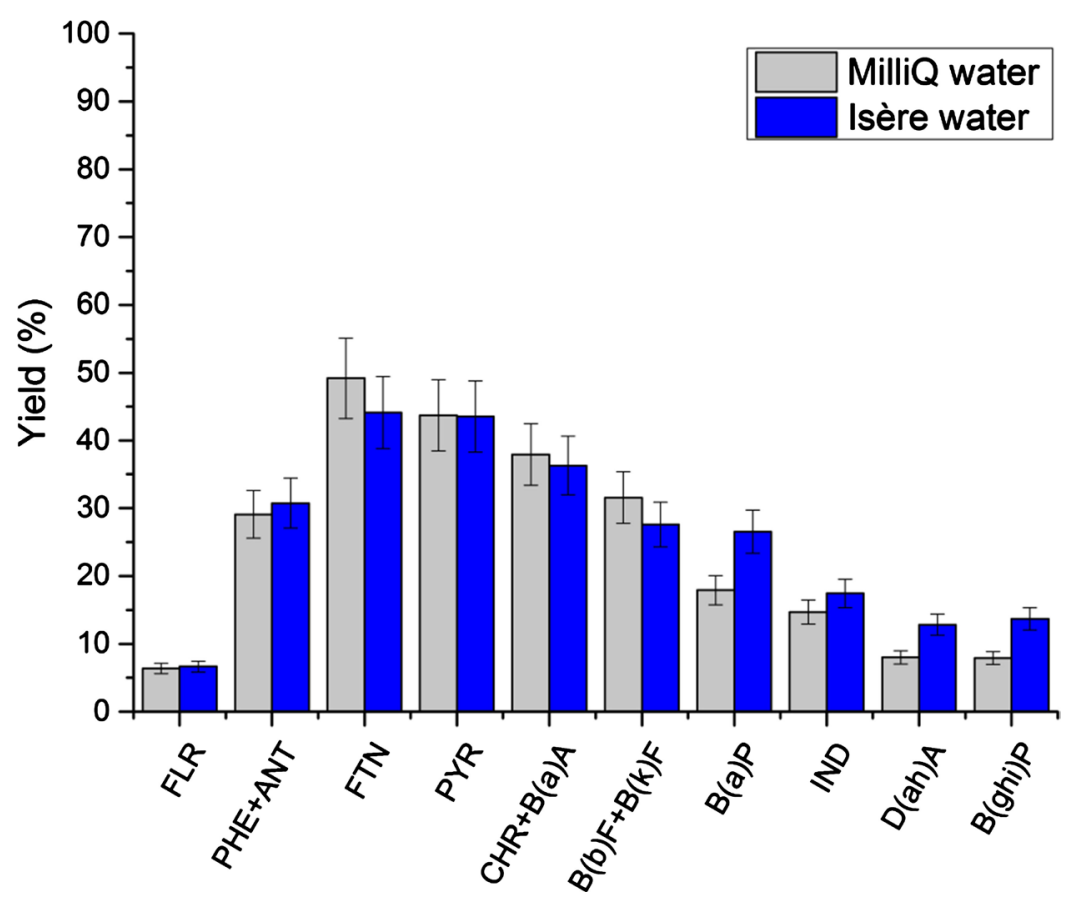

Figure 9. Comparison of the extraction yield obtained with $5 \mathrm{~mL}$ purified water or filtered natural water samples spiked in both case with the 16 PAHs at $5 \mu \mathrm{g} / \mathrm{L}$ (error bars were deduced from the standard deviations in Figure 8).

compared to other extraction techniques has the advantage of reduced foot print, reduced process time, reduced energy consumption as well as no solvent use. Repeatable recovery yields have been determined for $1 \mu \mathrm{g} / \mathrm{L}$ spiked samples and the analysis of PAHs in a natural water sample has been demonstrated without loss of performance compared to purified water samples. The results validate the use of our system for performing combined extraction and thermal desorption of PAHs contained in water samples. Thus our prototype is adapted to realize continuously the analysis of PAHs in an automatic and reproducible way.

Further works should be done to improve the extraction recovery of volatile PAHs or to study the extraction of other organic compounds in aim to develop a multi-residue extraction technique. Besides, these results paves the way for further integration and development of a fully portable analytical system by coupling the present system to miniaturize GC system for real-time in-situ PAHs monitoring in environmental samples like natural water samples. Such a system would be very effective for real-time monitoring of PAHs, for instance by sending a warning when a concentration threshold is exceeded.

\section{Acknowledgements}

This work was supported by the French National Research Agency (ANR) through Carnot funding. We acknowledge Thomas Bordy and Raymond Charles for their help in the microchip heating socket design, Louise Foan for preliminary extraction tests, Benoit Brevard for Labview program development, Manuel 
Alessio for chip packaging and Amélie Bellemin-Comte for microtechnology support within the CEA.

\section{Conflicts of Interest}

The authors declare no conflicts of interest regarding the publication of this paper.

\section{References}

[1] International Agency for Research of Cancer (2010) Some Non-Heterocyclic Polycyclic Aromatic Hydrocarbons and Some Related Exposures. IARC Monographs on the Evaluation of Carcinogenic Risks to Humans. Vol. 92, Lyon.

[2] U. EPA (2014) Polycyclic Aromatic Hydrocarbons (PAHs). United States Environmental Protection Agency, Washington DC.

[3] European Parliament (2013) Directive 2013/39/EU of the European Parliament and of the Council of 12 August 2013 Amending Directives 2000/60/EC and 2008/105/EC as Regards Priority Substances in the Field of Water Policy.

[4] Konieczka, P., Wolska, L. and Namieśnik, J. (2010) Quality Problems in Determination of Organic Compounds in Environmental Samples, Such as PAHs and PCBs. Trends in Analytical Chemistry, 29, 706-717. https://doi.org/10.1016/j.trac.2010.03.012

[5] Kumar, S., Negi, S. and Maiti, P. (2017) Biological and Analytical Techniques Used for Detection of Polyaromatic Hydrocarbons. Environmental Science and Pollution Research, 24, 25810-25827. https://doi.org/10.1007/s11356-017-0415-2

[6] Popp, P., Bauer, C. and Wennrich, L. (2001) Application of Stir Bar Sorptive Extraction in Combination with Column Liquid Chromatography for the Determination of Polycyclic Aromatic Hydrocarbons in Water Samples. Analytica Chimica Acta, 436, 1-9. https://doi.org/10.1016/S0003-2670(01)00895-9

[7] Ouyang, G.F. and Pawliszyn, J. (2006) Recent Developments in SPME for On-Site Analysis and Monitoring. TrAC Trends in Analytical Chemistry, 25, 692-703. https://doi.org/10.1016/j.trac.2006.05.005

[8] Plotka-Wasylka, J., et al. (2016) Modern Trends in Solid Phase Extraction: New Sorbent Media. TrAC Trends in Analytical Chemistry, 77, 23-43.

https://doi.org/10.1016/j.trac.2015.10.010

[9] Wen, Y.Y., et al. (2014) Recent Advances in Solid-Phase Sorbents for Sample Preparation Prior to Chromatographic Analysis. TrAC Trends in Analytical Chemistry, 59, 26-41. https://doi.org/10.1016/j.trac.2014.03.011

[10] Avino, P., et al. (2017) New Protocol Based on High-Volume Sampling Followed by DLLME-GC-IT/MS for Determining PAHs at Ultra-Trace Levels in Surface Water Samples. Microchemical Journal, 133, 251-257. https://doi.org/10.1016/j.microc.2017.03.052

[11] Fernandez-Amado, M., et al. (2016) A Novel and Cost-Effective Method for the Determination of Fifteen Polycyclic Aromatic Hydrocarbons in Low Volume Rainwater Samples. Talanta, 155, 175-184. https://doi.org/10.1016/j.talanta.2016.04.032

[12] Liew, C.S.M., Lo, X. and Lee, H.K. (2016) Miniscale Liquid-Liquid Extraction Coupled with Full Evaporation Dynamic Headspace Extraction for the Gas Chromatography/Mass Spectrometric Analysis of Polycyclic Aromatic Hydrocarbons with 4000-to-14000-Fold Enrichment. Analytical Chemistry, 88, 9095-9102. https://doi.org/10.1021/acs.analchem.6b02056 
[13] Zhang, M., et al. (2017) Portable Kit for High-Throughput Analysis of Polycyclic Aromatic Hydrocarbons Using Surface Enhanced Raman Scattering after Dispersive Liquid-Liquid Microextraction. Talanta, 175, 495-500. https://doi.org/10.1016/j.talanta.2017.07.072

[14] Yan, J., et al. (2018) Determination of Polycyclic Aromatic Hydrocarbons in Surface Water Using Simplified Liquid-Liquid Micro-Extraction and Pseudo-MRM GC/ MS/MS. Analytical Methods, 10, 405-416. https://doi.org/10.1039/C7AY01902E

[15] Wolska, L., Rawa-Adkonis, M. and Namiesnik, J. (2005) Determining PAHs and PCBs in Aqueous Samples: Finding and Evaluating Sources of Error. Analytical and Bioanalytical Chemistry, 382, 1389-1397. https://doi.org/10.1007/s00216-005-3280-7

[16] Fu, L.M., et al. (2018) Sample Preconcentration from Dilute Solutions on Micro/ Nanofluidic Platforms: A Review. Electrophoresis, 39, 289-310. https://doi.org/10.1002/elps.201700340

[17] Lafleur, J.P., et al. (2010) Miniaturised Centrifugal Solid Phase Extraction Platforms for In-Field Sampling, Pre-Concentration and Spectrometric Detection of Organic Pollutants in Aqueous Samples. Talanta, 81, 722-726. https://doi.org/10.1016/j.talanta.2009.12.001

[18] Duffy, E., et al. (2017) New Strategies for Stationary Phase Integration within Centrifugal Microfluidic Platforms for Applications in Sample Preparation and Pre-Concentration. Analytical Methods, 9, 1998-2006. https://doi.org/10.1039/C7AY00127D

[19] Peroni, D., et al. (2012) Advancing Liquid/Liquid Extraction through a Novel Microfluidic Device: Theory, Instrumentation and Applications in Gas Chromatography. Journal of Chromatography $A, 1226,77-86$. https://doi.org/10.1016/j.chroma.2011.08.001

[20] Ramos-Payan, M., Maspoch, S. and Llobera, A. (2017) A Simple and Fast Double-Flow Microfluidic Device Based Liquid-Phase Microextraction, (DF-mu LPME) for the Determination of Parabens in Water Samples. Talanta, 165, 496-501. https://doi.org/10.1016/j.talanta.2016.12.059

[21] Foan, L., Ricoul, F. and Vignoud, S. (2015) A Novel Microfluidic Device for Fast Extraction of Polycyclic Aromatic Hydrocarbons (PAHs) from Environmental Waters-Comparison with Stir-Bar Sorptive Extraction (SBSE). International Journal of Environmental Analytical Chemistry, 95, 1171-1185. https://doi.org/10.1080/03067319.2014.994617

[22] Foan, L., et al. (2018) Development of a New Phase for Lab-on-a-Chip Extraction of Polycyclic Aromatic Hydrocarbons from Water. Sensors and Actuators B-Chemical, 255, 1039-1047. https://doi.org/10.1016/j.snb.2017.08.151

[23] Camara, E.H.M., et al. (2010) Micro Gas Preconcentrator in Porous Silicon Filled with a Carbon Absorbent. Sensors and Actuators B-Chemical, 148, 610-619. https://doi.org/10.1016/j.snb.2010.05.054

[24] Haudebourg, R., et al. (2013) Temperature-Programmed Sputtered Micromachined Gas Chromatography Columns: An Approach to Fast Separations in Oilfield Applications. Analytical Chemistry, 85, 114-120. https://doi.org/10.1021/ac3022136

[25] McCartney, M.M., et al. (2017) An Easy to Manufacture Micro Gas Preconcentrator for Chemical Sensing Applications. ACS Sensors, 2, 1167-1174. https://doi.org/10.1021/acssensors.7b00289

[26] Reidy, S., et al. (2007) Temperature-Programmed GC Using Silicon Microfabricated Columns with Integrated Heaters and Temperature Sensors. Analytical Chemistry, 
79, 2911-2917. https://doi.org/10.1021/ac062148s

[27] Mery, E., et al. (2008) A Silicon Microfluidic Chip Integrating an Ordered Micropillar Array Separation Column and a Nano-Electrospray Emitter for LC/MS Analysis of Peptides. Sensors and Actuators B-Chemical, 134, 438-446.

https://doi.org/10.1016/j.snb.2008.05.037

[28] Arias-Estevez, M., et al. (2007) Sorption of PAHs to Colloid Dispersions of Humic Substances in Water. Bulletin of Environmental Contamination and Toxicology, 79, 251-254. https://doi.org/10.1007/s00128-007-9022-0

[29] Xia, X.H., Zhai, Y.W. and Dong, J.W. (2013) Contribution Ratio of Freely to Total Dissolved Concentrations of Polycyclic Aromatic Hydrocarbons in Natural River Waters. Chemosphere, 90, 1785-1793.

https://doi.org/10.1016/j.chemosphere.2012.08.021 


\section{Supplementary Material}

Table S1. Compounds studied, acronym, numbers of rings, water-octanol partition coefficients $(\log \mathrm{Ko} / \mathrm{w})$, molecular weight $(\mathrm{g} / \mathrm{mol})$.

\begin{tabular}{ccccc}
\hline Compound & Acronym & $\begin{array}{c}\text { Number } \\
\text { of rings }\end{array}$ & Log Ko/ $\mathrm{w}^{\mathrm{a}}$ & $\begin{array}{c}\text { Molecular Weight } \\
(\mathrm{g} / \mathrm{mol})\end{array}$ \\
\hline Naphthalene & NAP & 2 & 3.3 & 128 \\
Acenaphtylene & ACL & 3 & 3.94 & 152 \\
Acenaphthene & ACE & 3 & 3.92 & 154 \\
Fluorene & FLR & 3 & 4.18 & 165 \\
Phenantrene & PHE & 3 & 4.46 & 178 \\
Anthracene & ANT & 3 & 4.45 & 178 \\
Fluoranthene & FTN & 4 & 5.16 & 202 \\
Pyrene & PYR & 4 & 4.88 & 202 \\
Benzo[a]anthracene & B(a)A & 4 & 5.66 & 228 \\
Chrysene & CHR & 4 & 5.81 & 228 \\
Benzo[b]fluoranthene & B(b)F & 5 & 5.78 & 252 \\
Benzo[k]fluoranthene & B(k)F & 5 & 6.11 & 252 \\
Benzo[a]pyrene & B(a)P & 5 & 6.13 & 252 \\
Indeno[1, 2, 3-c, d]pyrene & IND & 5 & 6.75 & 278 \\
Benzo[g, h, i]perylene & B(ghi)P & 6 & 6.63 & 276 \\
Dibenzo[a, h]anthracene & D(ah)A & 6 & 6.7 & 276 \\
\hline
\end{tabular}

${ }^{\mathrm{a} O c t a n o l / w a t e r ~ p a r t i t i o n ~ c o e f f i c i e n t ~ a r e ~ g i v e n ~ i n ~ S a n c h e z-A v i l a, ~ J . ; ~ Q u i n t a n a, ~ J . ; ~ V e n t u r a, ~ F . ; ~ T a u l e r, ~ R . ; ~}$ Duarte, C. M.; Lacorte, S. Mar Pollut Bull 2010, 60, (1), 103-112.

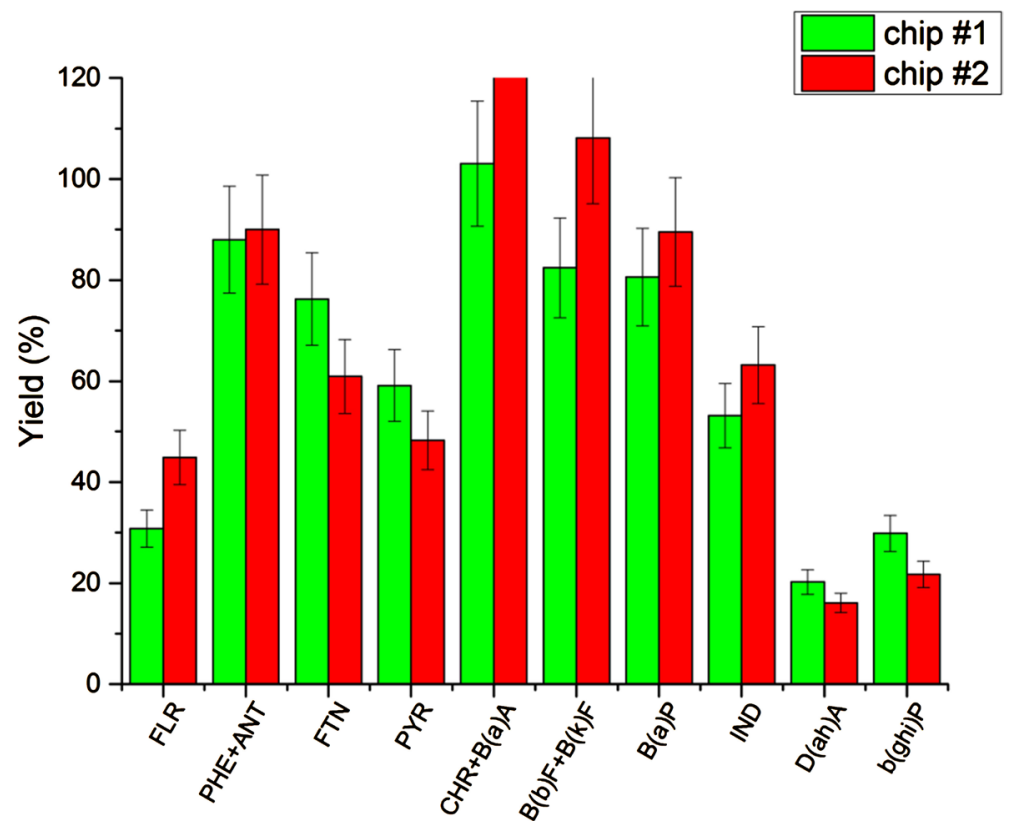

Figure S1. Comparison of the recovery yields obtained with 2 different chips for the extraction of $10 \mathrm{~mL}$ purified water sample spiked with the $16 \mathrm{PAHs}$ at $1 \mu \mathrm{g} / \mathrm{L}$ (error bars represent the standard deviation for $\mathrm{n}=4$ measures). 